\title{
Passivity and Synchronization of Multiple Multi-Delayed Neural Networks via Impulsive Control
}

\author{
Yong Wang, ${ }^{1}$ Zhichun Yang $\mathbb{D}^{2,3}{ }^{2,3}$ Tonglai Liu, ${ }^{1}$ and Hong-An Tang $\mathbb{D}^{2,4,5}$ \\ ${ }^{1}$ School of Computers, Guangdong University of Technology, Guangzhou 510006, China \\ ${ }^{2}$ Chongqing Key Lab on IFBDA, School of Mathematical Sciences, Chongqing Normal University, Chongqing 400047, China \\ ${ }^{3}$ School of Mathematical Sciences, Chongqing Normal University, Chongqing 400047, China \\ ${ }^{4}$ School of Computer Science and Technology, Tiangong University, Tianjin 300387, China \\ ${ }^{5}$ School of Artificial Intelligence, Chongqing University of Technology, Chongqing 401135, China \\ Correspondence should be addressed to Hong-An Tang; tanghongan163@163.com
}

Received 12 May 2020; Accepted 26 June 2020; Published 18 July 2020

Guest Editor: Hao Shen

Copyright (C) 2020 Yong Wang et al. This is an open access article distributed under the Creative Commons Attribution License, which permits unrestricted use, distribution, and reproduction in any medium, provided the original work is properly cited.

This paper is concerned with the passivity and synchronization for multiple multi-delayed neural networks (MMDNNs) under impulsive control. To ensure the passivity, input strict passivity, and output strict passivity in MMDNNs, a suitable impulsive controller is designed. Moreover, an impulsive time-dependent Lyapunov functional is exploited to obtain the synchronization criterion of MMDNNs, where the criterion is formulated by linear matrix inequalities. Numerical examples are given to verify the validity of the theoretical results.

\section{Introduction}

Neural networks (NNs) have received extensive attention due to their successful applications in vision system [1], associative memory [2], pattern recognition [3], and image compression [4]. NNs always require stability, which is a prerequisite for many applications. Therefore, the stability of NNs has become a hot issue in recent years [5-11]. Zhang et al. [7] considered some asymptotic stability criteria of NNs with distributed delays based on Lyapunov-Krasovskii functionals. By improving the auxiliary polynomial-based functions, Li et al. [9] solved the stability problem in delayed NNs. Since the main property of passivity is to keep the system internally stable, some researchers have focused on the passivity for NNs [12-17]. Lian et al. [14] proposed a kind of switched NNs with time-varying delays and stochastic disturbances, and the passivity of networks was analyzed by designing a state-dependent switching law and a hysteresis switching law. Cao et al. [17] addressed the robust passivity issue of uncertain NNs with additive time-varying delays and leakage delay, and a general activation function was utilized to ensure that the proposed network model was passive.
In addition, multi-weighted network models [18-21] can be used to describe many real-world networks including public transportation road networks, communication networks, social networks, and so forth. Recently, some researchers have investigated the dynamical behaviors of complex networks with multiple weights [20,21]. Wang et al. [20] concentrated on two types of multi-weighted complex networks with several different weights between two nodes, and sufficient conditions ensuring the synchronization were developed by utilizing the pinning control method. Under the help of pinning adaptive control techniques, the passivity of multi-weighted complex networks with different dimensions of input and output was discussed in [21]. However, a few authors have considered the stability and passivity of NNs with multiple delays.

Compared with continuous control, there are many advantages of impulsive control strategies, which include low maintenance costs, high reliability, ease of installation, and high efficiency [22]. So far, a series of investigations in regard to the stability [23-28] and passivity [29-31] for impulsive NNs have been reported. Zhu and Cao [25] dealt with the stability problem of impulsive stochastic BAM NNs 
with mixed time delays and Markovian jump parameters by exploiting Itô's formula and stochastic analysis theory. According to the comparison principle and compression mapping theorem, the global exponential stability of periodic solution was considered in an array of CohenGrossberg NNs with time-varying delays and periodic coefficients via impulsive control in [26]. Zhou [31] took into account the passivity of recurrent NNs with multiproportional delays and impulse. But very few authors have discussed the stability and passivity problems of multi-delayed NNs under impulsive control.

At present, most of the literatures with respect to identifying network structures from the observation and control of dynamical behavior are concentrated in a single neural network $[5,7,12,13]$. In practical applications, some tasks are difficult to complete by a single neural network; even if the network can accomplish these tasks, it may result in high costs. But multiple NNs can solve some difficult problems through cooperation with each other so that the cost can be reduced. Recently, some cooperative control problems [32-35] involving passivity and synchronization [36-38] have been concerned in multiple NNs. Unfortunately, as far as we know, the passivity and synchronization of multiple multi-delayed NNs (MMDNNs) via impulsive control have never been considered. Inspired by the above discussion, this paper aims to further study the passivity and global exponential synchronization of MMDNNs by using impulsive control techniques. The contributions of this paper are as follows. First, compared with the traditional impulse-time-independent Lyapunov functional, the impulse-time-dependent feature of the Lyapunov functional in this paper can capture more dynamical behaviors of MMDNNs. Second, with the help of some piecewise linear functions and inequality techniques, the passivity problems of MMDNNs are addressed via impulsive control. Third, a newly designed impulsive controller is applied to synchronize the proposed networks.

\section{Preliminaries}

2.1. Notations. Let $\mathcal{N}=\{1,2, \ldots, N\}, \mathbb{N}=\{0,1,2, \ldots\}$. The fixed moments $t_{k}$ satisfy $0=t_{0}<t_{1}<t_{2}<\cdots<t_{k}<\cdots$ and $\lim _{k \longrightarrow+\infty} t_{k}=+\infty, k \in \mathbb{N} . \quad \lambda_{\min }(\cdot)\left(\lambda_{\max }(\cdot)\right)$ denotes the smallest (largest) eigenvalue of a matrix. For any $\xi(t)=$ $\left(\xi_{1}(t), \xi_{2}(t), \ldots, \xi_{N}(t)\right)^{T} \in \mathbb{R}^{N}, \quad\|\xi(t)\|=\left(\sum_{i=1}^{N} \xi_{i}^{2}(t)\right)^{1 / 2}$, $\|\xi(t)\|_{\tau}=\sup _{-\tau \leq \epsilon \leq 0}\|\xi(t+\epsilon)\|$.

The notation $\mathbb{S}\left(v_{1}, v_{2}\right)$ represents the set of impulse time sequences $\left\{t_{k}\right\}$ satisfying $\nu_{2} \geq t_{k+1}-t_{k} \geq v_{1}>0$ for all $k \in \mathbb{N}$, in which $v_{1}, v_{2} \in \mathbb{R}$. For a given impulse time sequence $\left\{t_{k}\right\} \in \mathbb{S}\left(v_{1}, v_{2}\right)$, some piecewise linear functions can be defined as follows:

$$
\begin{gathered}
\phi(t)=\frac{1}{t_{k+1}-t_{k}}, \quad t \in\left[t_{k}, t_{k+1}\right), k \in \mathbb{N}, \\
\phi_{1}(t)= \begin{cases}\left(t-t_{k}\right) \phi(t), & t \in\left[t_{k}, t_{k+1}\right), k \in \mathbb{N}, \\
1, & t_{0}>t .\end{cases}
\end{gathered}
$$

Let $\phi_{2}(t)=1-\phi_{1}(t)$. It is obvious to see that $[0,1] \ni \phi_{1}(t), \quad[0,1] \ni \phi_{2}(t)$, for $\mathbb{R} \ni t$, and $\phi_{1}\left(t_{k}\right)=$ $\phi_{2}\left(t_{k}^{-}\right)=0, \phi_{1}\left(t_{k}^{-}\right)=\phi_{2}\left(t_{k}\right)=1, \mathbb{N} \ni k$.

For $\left\{t_{k}\right\} \in \mathbb{S}\left(v_{1}, v_{2}\right), \phi(t)$ can be written as

$$
\phi(t)=\frac{\psi_{1}(t)}{v_{1}}+\frac{\psi_{2}(t)}{v_{2}}
$$

where

$$
\begin{aligned}
& \psi_{1}(t)=1-\psi_{2}(t), \\
& \psi_{2}(t)= \begin{cases}\frac{\phi(t)-\left(1 / \nu_{2}\right)}{\left(1 / \nu_{1}\right)-\left(1 / \nu_{2}\right)}, & \text { if } v_{1} \neq v_{2}, \\
1, & \text { if } v_{1}=v_{2} .\end{cases}
\end{aligned}
$$

Definition 1 (see [21]). A system is said to be passive with output $y(t) \in \mathbb{R}^{\epsilon N}$ and input $v(t) \in \mathbb{R}^{n N}$, if there is a storage function $S:[0,+\infty) \longrightarrow[0,+\infty)$ and a matrix $A \in \mathbb{R}^{\epsilon N \times n N}$ satisfying

$$
S\left(t_{\varrho}\right)-S\left(t_{0}\right) \leq \int_{t_{0}}^{t_{\varrho}} y^{T}(t) A v(t) \mathrm{d} t,
$$

for any $t_{\varrho}, t_{0} \in[0,+\infty)$ and $t_{0} \leq t_{\varrho}$.

Definition 2 (see [21]). A system is said to be strictly passive with output $y(t) \in \mathbb{R}^{\epsilon N}$ and input $v(t) \in \mathbb{R}^{n N}$, if there is a storage function $S:[0,+\infty) \longrightarrow[0,+\infty)$, matrices $A \in \mathbb{R}^{\epsilon N \times n N}, 0 \leq A_{1} \in \mathbb{R}^{n N \times n N}, 0 \leq A_{2} \in \mathbb{R}^{\epsilon N \times \epsilon N}$, and eigenvalues $\lambda_{m}\left(A_{1}\right)+\lambda_{m}\left(A_{2}\right)>0$ satisfying

$$
\begin{aligned}
S\left(t_{\varrho}\right)-S\left(t_{0}\right) \leq & \int_{t_{0}}^{t_{\mathrm{e}}}\left[y^{T}(t) A v(t)-v^{T}(t) A_{1} v(t)\right. \\
& \left.-y^{T}(t) A_{2} y(t)\right] \mathrm{d} t,
\end{aligned}
$$

for any $t_{\varrho}, t_{0} \in[0,+\infty)$ and $t_{0} \leq t_{\varrho}$.

The system is input-strictly passive if $0<A_{1}$ and outputstrictly passive if $0<A_{2}$.

\section{Passivity of MMDNNs via Impulsive Control}

3.1. Network Model. The MMDNNs are considered as follows:

$$
\dot{z}_{i}(t)=-D z_{i}(t)+\sum_{\imath=1}^{p} W_{\iota} g^{\imath}\left(z_{i}\left(t-\tau_{\iota}\right)\right)+J+v_{i}(t),
$$

in which $i \in \mathcal{N} ; z_{i}(t)=\left(z_{i 1}(t), z_{i 2}(t), \ldots, z_{\text {in }}(t)\right)^{T} \in \mathbb{R}^{n}$ is the state vector of node $i ; D=\operatorname{diag}\left(d_{1}, d_{2}, \ldots, d_{n}\right)>0$; $W_{\iota} \in \mathbb{R}^{n \times n} ; g^{l}\left(z_{i}\left(t-\tau_{\iota}\right)\right)=\left(g_{1}^{l}\left(z_{i 1}\left(t-\tau_{\iota}\right)\right), g_{2}^{l}\left(z_{i 2}\left(t-\tau_{\iota}\right)\right)\right.$, $\left.\ldots, g_{n}^{l}\left(z_{\text {in }}\left(t-\tau_{l}\right)\right)\right)^{T} \in \mathbb{R}^{n} ; J=\left(J_{1}, J_{2}, \ldots, J_{n}\right)^{T} \in \mathbb{R}^{n} ; v_{i}(t) \in$ $\mathbb{R}^{n}$ means the input vector of node $i$; and $\tau_{l}$ is the transmission delay and $0 \leq \tau_{\iota} \leq \tau$.

In this paper, there is $\mathbb{R} \ni \rho_{\varsigma}>0(\varsigma=1,2, \ldots, n)$ such that

$$
\left|g_{\zeta}^{\iota}\left(\theta_{1}\right)-g_{\varsigma}^{\iota}\left(\theta_{2}\right)\right| \leq \rho_{\varsigma}^{\iota}\left|\theta_{1}-\theta_{2}\right|
$$

for any $\theta_{1}, \theta_{2} \in \mathbb{R}$. Let $\Lambda_{l}=\operatorname{diag}\left(\left(\rho_{1}^{l}\right)^{2},\left(\rho_{2}^{l}\right)^{2}, \ldots,\left(\rho_{n}^{l}\right)^{2}\right)$. 
Suppose that $z^{*}=\left(z_{1}^{*}, z_{2}^{*}, \ldots, z_{n}^{*}\right)^{T} \in \mathbb{R}^{n}$ is an equilibrium solution of an isolated node of the MMDNNs (6). Then, one gets

$$
-D z^{*}+J+\sum_{\imath=1}^{p} W_{\iota} g^{\iota}\left(z^{*}\right)=0 .
$$

For the MMDNNs (6), construct the following impulsive controller:

$$
u_{i}\left(t_{k}\right)=\sum_{j=1}^{N} B_{i j} \Phi z_{j}\left(t_{k}^{-}\right), \quad k \in \mathbb{N}, i \in \mathcal{N},
$$

in which $0<\Phi \in \mathbb{R}^{n \times n} ; z_{i}\left(t_{k}^{-}\right)=\lim _{t \longrightarrow t_{t}} z_{i}(t) ; B=\left(B_{i j}\right)_{N \times N}$ means the impulsive coupling matrix, where $B_{i j}$ is described as follows: if there is a link from node $i$ to node $j$, then ; otherwise, $B_{i j}=0(i \neq j)$; and

$$
B_{i i}=-\sum_{\substack{j=1 \\ j \neq i}}^{N} B_{i j}, \quad i \in \mathcal{N} .
$$

It is derived from (6) and (9) that

$$
\left\{\begin{array}{l}
\dot{z}_{i}(t)=-D z_{i}(t)+\sum_{i=1}^{p} W_{\iota} g^{l}\left(z_{i}\left(t-\tau_{\iota}\right)\right)+J+v_{i}(t), \quad t \neq t_{k}, \\
I_{k}\left(z_{i}(t)\right)=\sum_{j=1}^{N} B_{i j} \Phi z_{j}\left(t_{k}^{-}\right), \quad k \in \mathbb{N}, i \in \mathcal{N},
\end{array}\right.
$$

where $I_{k}\left(z_{i}(t)\right)=z_{i}\left(t_{k}^{+}\right)-z_{i}\left(t_{k}^{-}\right), \quad z_{i}\left(t_{k}^{+}\right)=\lim _{t \longrightarrow t_{k}^{+}} z_{i}(t)$. Suppose $z_{i}\left(t_{k}\right)=z_{i}\left(t_{k}^{+}\right)$.

Let $\zeta_{i}(t)=z_{i}(t)-z^{*}$. Then, by (8) and (11), we acquire

$$
\left\{\begin{array}{l}
\dot{\zeta}_{i}(t)=-D \zeta_{i}(t)+\sum_{i=1}^{p} W_{\iota}\left[g^{t}\left(z_{i}\left(t-\tau_{\iota}\right)\right)-g^{\iota}\left(z^{*}\right)\right]+v_{i}(t), \quad t \neq t_{k}, \\
I_{k}\left(\zeta_{i}(t)\right)=\sum_{j=1}^{N} B_{i j} \Phi \zeta_{j}\left(t_{k}^{-}\right), \quad k \in \mathbb{N}, i \in \mathcal{N} .
\end{array}\right.
$$

The output vector $y_{i}(t) \in \mathbb{R}^{\epsilon}$ of the MMDNNs (12) is chosen as

$$
y_{i}(t)=C_{1} \zeta_{i}(t)+C_{2} v_{i}(t),
$$

in which $C_{1} \in \mathbb{R}^{e \times n}$ and $C_{2} \in \mathbb{R}^{e \times n}$ are known matrices.

\subsection{Passivity Criteria}

Theorem 1. Under the impulsive controller (9), the MMDNNs (12) are passive over $\mathbb{S}\left(v_{1}, v_{2}\right)$ if there exist matrices $0<F_{1} \in \mathbb{R}^{n N \times n N}, 0<F_{2} \in \mathbb{R}^{n N \times n N}$, and $A \in \mathbb{R}^{e N \times n N}$ and a scalar $\gamma \in(0,1]$ such that

$$
\Gamma_{1 r h}=\left(\begin{array}{cc}
\Omega_{1 r h} & \Omega_{2 r} \\
\Omega_{2 r}^{T} & \Omega_{3}
\end{array}\right) \leq 0,
$$

$$
\left(\begin{array}{cc}
-\gamma F_{1} & \left(I_{n N}+B \otimes \Phi\right)^{T} F_{2} \\
F_{2}\left(I_{n N}+B \otimes \Phi\right) & -F_{2}
\end{array}\right) \leq 0,
$$

in which $r=1,2, h=1,2, \Omega_{1 r h}=1 / v_{h}\left(F_{1}-F_{2}\right)-F_{r}\left(I_{N} \otimes D\right)$ $-\left(I_{N} \otimes D\right) F_{r}+\sum_{l=1}^{p}\left\{F_{r}\left[I_{N} \otimes\left(W_{l} W_{l}^{T}\right)\right] F_{r}+I_{N} \otimes \Lambda_{l}\right\}, \quad \Omega_{2 r}=$ $F_{r}-\left(I_{N} \otimes C_{1}^{T}\right) A$, and $\Omega_{3}=-A^{T}\left(I_{N} \otimes C_{2}\right)-\left(I_{N} \otimes C_{2}^{T}\right) A$.

Proof. Let $F(t)=\sum_{r=1}^{2} \phi_{r}(t) F_{r}, \Omega_{1}(t)=\phi(t)\left(F_{1}-F_{2}\right)-\left(I_{N} \otimes\right.$ $D) F(t)-F(t)\left(I_{N} \otimes D\right)+\sum_{l=1}^{p}\left\{F(t)\left[I_{N} \otimes\left(W_{l} W_{l}^{T}\right)\right] F(t)+\right.$ $\left.I_{N} \otimes \Lambda_{\ell}\right\}, \Omega_{2}(t)=F(t)-\left(I_{N} \otimes C_{1}^{T}\right) A$.

Then, by (14), we have

$$
\Gamma_{1}(t) \triangleq \sum_{r, h=1}^{2} \phi_{r}(t) \psi_{h}(t) \Gamma_{1 r h}=\left(\begin{array}{cc}
\Omega_{1}(t) & \Omega_{2}(t) \\
\Omega_{2}^{T}(t) & \Omega_{3}
\end{array}\right) \leq 0 .
$$

The impulse-time-dependent Lyapunov functional for the MMDNNs (12) is considered as follows:

$$
V_{1}(t)=\zeta^{T}(t) F(t) \zeta(t)+\sum_{l=1}^{p} \int_{t-\tau_{\iota}}^{t} \zeta^{T}(s)\left(I_{N} \otimes \Lambda_{\iota}\right) \zeta(s) \mathrm{d} s,
$$

where $\zeta(t)=\left(\zeta_{1}^{T}(t), \zeta_{2}^{T}(t), \ldots, \zeta_{N}^{T}(t)\right)^{T}$.

Then,

$$
\begin{aligned}
\dot{V}_{1}(t)= & \zeta^{T}(t)\left[\phi(t)\left(F_{1}-F_{2}\right)-\left(I_{N} \otimes D\right) F(t)\right. \\
& \left.-F(t)\left(I_{N} \otimes D\right)\right] \zeta(t)+2 \zeta^{T}(t) F(t) \\
& \cdot\left\{\sum_{l=1}^{p}\left(I_{N} \otimes W_{\iota}\right) \times\left[\hat{g}^{l}\left(z\left(t-\tau_{\iota}\right)\right)-\hat{g}^{l}\left(\widehat{z}^{*}\right)\right]+v(t)\right\} \\
& -\sum_{\imath=1}^{p} \zeta^{T}\left(t-\tau_{\iota}\right)\left(I_{N} \otimes \Lambda_{\iota}\right) \zeta\left(t-\tau_{\iota}\right) \\
& +\sum_{l=1}^{p} \zeta^{T}(t)\left(I_{N} \otimes \Lambda_{\iota}\right) \zeta(t),
\end{aligned}
$$

where $\quad \hat{z}^{*}=\left(\left(z^{*}\right)^{T},\left(z^{*}\right)^{T}, \ldots,\left(z^{*}\right)^{T}\right)^{T}, \quad \hat{g}^{\prime}\left(z\left(t-\tau_{\iota}\right)\right)=$ $\left(\left(g^{l}\left(z_{1}\left(t-\tau_{\iota}\right)\right)\right)^{T},\left(g^{l}\left(z_{2}\left(t-\tau_{1}\right)\right)\right)^{T}, \ldots,\left(g^{l}\left(z_{N}\left(t-\tau_{\imath}\right)\right)\right)^{T}\right)^{T}$, $\widehat{g}^{l}\left(\widehat{z}^{*}\right)=\left(\left(g^{l}\left(z^{*}\right)\right)^{T},\left(g^{l}\left(z^{*}\right)\right)^{T}, \ldots, \quad\left(g^{l}\left(z^{*}\right)\right)^{T}\right)^{T}, v(t)=\left(v_{1}^{T}\right.$ $\left.(t), v_{2}^{T}(t), \ldots, v_{N}^{T}(t)\right)^{T}$.

Obviously,

$$
\begin{aligned}
& 2 \zeta^{T}(t) F(t)\left(I_{N} \otimes W_{\iota}\right)\left[\hat{g}^{\iota}\left(z\left(t-\tau_{\iota}\right)\right)-\hat{g}^{\iota}\left(\widehat{z}^{*}\right)\right] \\
& \quad \leq \zeta^{T}(t) F(t)\left[I_{N} \otimes\left(W_{\iota} W_{\iota}^{T}\right)\right] F(t) \zeta(t) \\
& \quad+\zeta^{T}\left(t-\tau_{\iota}\right)\left(I_{N} \otimes \Lambda_{\iota}\right) \zeta\left(t-\tau_{\iota}\right) .
\end{aligned}
$$

Substituting (19) into (18) yields

$$
\begin{aligned}
\dot{V}_{1}(t) \leq & \zeta^{T}(t)\left\{\phi(t)\left(F_{1}-F_{2}\right)-\left(I_{N} \otimes D\right) F(t)-F(t) \times\left(I_{N} \otimes D\right)+\sum_{\imath=1}^{p} F(t)\left[I_{N} \otimes\left(W_{\iota} W_{\imath}^{T}\right)\right] F(t)+\sum_{\imath=1}^{p} I_{N} \otimes \Lambda_{\iota}\right\} \zeta(t) \\
& +2 \zeta^{T}(t) F(t) v(t) .
\end{aligned}
$$


From (13), (16), and (20), one obtains

$$
\begin{aligned}
& \dot{V}_{1}(t)-2 y^{T}(t) A v(t) \\
& \quad \leq \zeta^{T}(t)\left\{\phi(t)\left(F_{1}-F_{2}\right)-\left(I_{N} \otimes D\right) F(t)-F(t)\left(I_{N} \otimes D\right)\right. \\
& \left.\quad+\sum_{\imath=1}^{p}\left\{F(t)\left[I_{N} \otimes\left(W_{\iota} W_{\iota}^{T}\right)\right] F(t)+I_{N} \otimes \Lambda_{\iota}\right\}\right\} \zeta(t) \\
& +v^{T}(t)\left[-A^{T}\left(I_{N} \otimes C_{2}\right)-\left(I_{N} \otimes C_{2}^{T}\right) A\right] v(t) \\
& +2 \zeta^{T}(t)\left[F(t)-\left(I_{N} \otimes C_{1}^{T}\right) A\right] v(t) \\
& \quad=\Phi_{1}^{T}(t)\left(\begin{array}{cc}
\Omega_{1}(t) & \Omega_{2}(t) \\
\Omega_{2}^{T}(t) & \Omega_{3}
\end{array}\right) \varpi_{1}(t) \\
& \quad \leq 0, t \in\left(t_{k}, t_{k+1}\right), \mathbb{N} \ni k,
\end{aligned}
$$

in which $\varpi_{1}(t)=\left(\zeta^{T}(t), v^{T}(t)\right)^{T}$.

Integrating (21) with respect to $t$ from $t_{0}$ to $t_{\mathrm{\rho}}\left(t_{\eta} \leq t_{\mathrm{\rho}}<t_{\eta+1}, \eta=0,1, \ldots\right)$, we acquire

$$
\begin{aligned}
& \int_{t_{0}}^{t_{\mathrm{e}}}\left[\dot{V}_{1}(t)-2 y^{T}(t) A v(t)\right] \mathrm{d} t \\
&=\int_{t_{\eta}}^{t_{\mathrm{e}}} \dot{V}_{1}(t) \mathrm{d} t+\sum_{\sigma=1}^{\eta} \int_{t_{\sigma-1}}^{t_{\sigma}} \dot{V}_{1}(t) \mathrm{d} t-2 \int_{t_{0}}^{t_{\mathrm{e}}} y^{T}(t) A v(t) \mathrm{d} t \\
&= {\left[V_{1}\left(t_{\varrho}\right)-V_{1}\left(t_{\eta}\right)\right]+\sum_{\sigma=1}^{\eta}\left[V_{1}\left(t_{\sigma}^{-}\right)-V_{1}\left(t_{\sigma-1}\right)\right] } \\
&-2 \int_{t_{0}}^{t_{\mathrm{\rho}}} y^{T}(t) A v(t) \mathrm{d} t \\
&= {\left[V_{1}\left(t_{\varrho}\right)-V_{1}\left(t_{0}\right)\right]+\sum_{\sigma=1}^{\eta}\left[V_{1}\left(t_{\sigma}^{-}\right)-V_{1}\left(t_{\sigma}\right)\right] } \\
&-2 \int_{t_{0}}^{t_{\mathrm{\rho}}} y^{T}(t) A v(t) \mathrm{d} t
\end{aligned}
$$

in which $t_{\rho}, t_{0} \in[0,+\infty)$ and $t_{0} \leq t_{\varrho}$.

At the impulse time $t_{k}, k \in \mathbb{N}$, according to the definition of $F(t)$, one gets

$$
\begin{aligned}
& F\left(t_{k}^{-}\right)=F_{1}, \\
& F\left(t_{k}\right)=F_{2} .
\end{aligned}
$$

It is found from (12), (15), and (17) that

$$
\begin{aligned}
V_{1}\left(t_{k}\right)= & \zeta^{T}\left(t_{k}\right) F\left(t_{k}\right) \zeta\left(t_{k}\right)+\sum_{l=1}^{p} \int_{t_{k}-\tau_{\iota}}^{t_{k}} \zeta^{T}(s)\left(I_{N} \otimes \Lambda_{l}\right) \zeta(s) \mathrm{d} s \\
= & \zeta^{T}\left(t_{k}^{-}\right)\left(I_{n N}+B \otimes \Phi\right)^{T} F_{2}\left(I_{n N}+B \otimes \Phi\right) \zeta\left(t_{k}^{-}\right) \\
& +\sum_{\imath=1}^{p} \int_{t_{k}-\tau_{l}}^{t_{k}} \zeta^{T}(s)\left(I_{N} \otimes \Lambda_{l}\right) \zeta(s) \mathrm{d} s \\
\leq & \gamma \zeta^{T}\left(t_{k}^{-}\right) F_{1} \zeta\left(t_{k}^{-}\right)+\sum_{\imath=1}^{p} \int_{t_{k}-\tau_{\iota}}^{t_{k}} \zeta^{T}(s)\left(I_{N} \otimes \Lambda_{\iota}\right) \zeta(s) \mathrm{d} s \\
\leq & \zeta^{T}\left(t_{k}^{-}\right) F\left(t_{k}^{-}\right) \zeta\left(t_{k}^{-}\right)+\sum_{l=1}^{p} \int_{t_{k}-\tau_{\iota}}^{t_{k}} \zeta^{T}(s)\left(I_{N} \otimes \Lambda_{\iota}\right) \zeta(s) \mathrm{d} s \\
= & V_{1}\left(t_{k}^{-}\right), \mathbb{N} \ni k .
\end{aligned}
$$

By (21)-(24), we have

$$
V_{1}\left(t_{\varrho}\right)-V_{1}\left(t_{0}\right) \leq 2 \int_{t_{0}}^{t_{\varrho}} y^{T}(t) A v(t) \mathrm{d} t .
$$

Hence,

$$
S\left(t_{\varrho}\right)-S\left(t_{0}\right) \leq \int_{t_{0}}^{t_{\varrho}} y^{T}(t) A v(t) \mathrm{d} t
$$

for any $t_{\varrho}, t_{0} \in[0,+\infty)$ and $t_{0} \leq t_{\varrho}$, in which $S(t)=V_{1}(t) / 2$.

Theorem 2. Under the impulsive controller (9), the MMDNNs (12) are input-strictly passive over $\mathbb{S}\left(v_{1}, v_{2}\right)$ if there exist matrices $0<F_{1} \in \mathbb{R}^{n N \times n N}, \quad 0<F_{2} \in \mathbb{R}^{n N \times n N}$, $A \in \mathbb{R}^{\epsilon N \times n N}$, and $0<A_{1} \in \mathbb{R}^{n N \times n N}$ and a scalar $\gamma \in(0,1]$ such that

$$
\begin{gathered}
\Gamma_{2 r h}=\left(\begin{array}{cc}
\Omega_{1 r h} & \Omega_{2 r} \\
\Omega_{2 r}^{T} & \Omega_{4}
\end{array}\right) \leq 0, \\
\left(\begin{array}{cc}
-\gamma F_{1} & \left(I_{n N}+B \otimes \Phi\right)^{T} F_{2} \\
F_{2}\left(I_{n N}+B \otimes \Phi\right) & -F_{2}
\end{array}\right) \leq 0,
\end{gathered}
$$

in which $r=1,2, h=1,2, \Omega_{1 r h}=1 / \nu_{h}\left(F_{1}-F_{2}\right)-F_{r}\left(I_{N} \otimes D\right)-$ $\left(I_{N} \otimes D\right) F_{r}+\sum_{l=1}^{p}\left\{F_{r}\left[I_{N} \otimes\left(W_{l} W_{l}^{T}\right)\right] F_{r}+I_{N} \otimes \Lambda_{l}\right\}, \Omega_{2 r}=F_{r}-$ $\left(I_{N} \otimes C_{1}^{T}\right) A, \Omega_{4}=2 A_{1}-A^{T}\left(I_{N} \otimes C_{2}\right)-\left(I_{N} \otimes C_{2}^{T}\right) A$.

Proof. Let

$F(t)=\sum_{r=1}^{2} \phi_{r}(t) F_{r}, \Omega_{1}(t)=\phi(t)\left(F_{1}-F_{2}\right)-\left(I_{N} \otimes\right.$

D) $F(t)-F(t)\left(I_{N} \otimes D\right)+\sum_{l=1}^{p}\left\{F(t)\left[I_{N} \otimes\left(W_{l} W_{l}^{T}\right)\right] \quad F(t)+\right.$ $\left.I_{N} \otimes \Lambda_{l}\right\}, \Omega_{2}(t)=F(t)-\left(I_{N} \otimes C_{1}^{T}\right) A$.

Then, from (27), one obtains

$$
\Gamma_{2}(t) \triangleq \sum_{r, h=1}^{2} \phi_{r}(t) \psi_{h}(t) \Gamma_{2 r h}=\left(\begin{array}{cc}
\Omega_{1}(t) & \Omega_{2}(t) \\
\Omega_{2}^{T}(t) & \Omega_{4}
\end{array}\right) \leq 0 .
$$

Selecting the same $V_{1}$ as (17) for the networks (12) and using (29), one has

$$
\begin{aligned}
\dot{V}_{1}(t) & -2 y^{T}(t) A v(t)+2 v^{T}(t) A_{1} v(t) \\
\leq & \zeta^{T}(t)\left\{\phi(t)\left(F_{1}-F_{2}\right)-\left(I_{N} \otimes D\right) F(t)-F(t)\left(I_{N} \otimes D\right)\right. \\
& \left.+\sum_{\imath=1}^{p}\left\{F(t)\left[I_{N} \otimes\left(W_{\iota} W_{\imath}^{T}\right)\right] F(t)+I_{N} \otimes \Lambda_{\iota}\right\}\right\} \zeta(t) \\
& +v^{T}(t)\left[2 A_{1}-A^{T}\left(I_{N} \otimes C_{2}\right)-\left(I_{N} \otimes C_{2}^{T}\right) A\right] v(t) \\
& +2 \zeta^{T}(t)\left[F(t)-\left(I_{N} \otimes C_{1}^{T}\right) A\right] v(t) \\
= & \Phi_{1}^{T}(t)\left(\begin{array}{l}
\Omega_{1}(t) \quad \Omega_{2}(t) \\
\Omega_{2}^{T}(t) \quad \Omega_{4}
\end{array}\right) \varpi_{1}(t) \\
\leq & 0, t \in\left(t_{k}, t_{k+1}\right), \mathbb{N} \ni k,
\end{aligned}
$$

in which $\oplus_{1}(t)=\left(\zeta^{T}(t), v^{T}(t)\right)^{T}$.

Integrating (30) with respect to $t$ from $t_{0}$ to $t_{\mathrm{Q}}\left(t_{\eta} \leq t_{\mathrm{Q}}<t_{\eta+1}, \eta=0,1, \ldots\right)$, we acquire 


$$
\begin{aligned}
\int_{t_{0}}^{t_{\mathrm{e}}} & {\left[\dot{V}_{1}(t)-2 y^{T}(t) A v(t)+2 v^{T}(t) A_{1} v(t)\right] \mathrm{d} t } \\
= & {\left[V_{1}\left(t_{\mathrm{e}}\right)-V_{1}\left(t_{0}\right)\right]+\sum_{\sigma=1}^{\eta}\left[V_{1}\left(t_{\sigma}^{-}\right)-V_{1}\left(t_{\sigma}\right)\right] } \\
& -2 \int_{t_{0}}^{t_{\mathrm{e}}}\left[y^{T}(t) A v(t) \mathrm{d} t-v^{T}(t) A_{1} v(t)\right] \mathrm{d} t,
\end{aligned}
$$

in which $t_{\mathrm{\rho}}, t_{0} \in[0,+\infty)$ and $t_{0} \leq t_{\mathrm{\rho}}$.

At the impulse time $t_{k}, k \in \mathbb{N}$, according to the definition of $F(t)$, one acquires

$$
\begin{aligned}
& F\left(t_{k}^{-}\right)=F_{1}, \\
& F\left(t_{k}\right)=F_{2} .
\end{aligned}
$$

On the basis of (12), (17), and (28), we get

$$
V_{1}\left(t_{k}\right) \leq V_{1}\left(t_{k}^{-}\right), \quad \mathbb{N} \ni k
$$

Considering (30)-(33), it is obtained that

$$
V_{1}\left(t_{\varrho}\right)-V_{1}\left(t_{0}\right) \leq 2 \int_{t_{0}}^{t_{\varrho}}\left[y^{T}(t) A v(t)-v^{T}(t) A_{1} v(t)\right] \mathrm{d} t .
$$

Thus,

$$
S\left(t_{\mathrm{e}}\right)-S\left(t_{0}\right) \leq \int_{t_{0}}^{t_{\mathrm{e}}} y^{T}(t) A v(t) \mathrm{d} t-\int_{t_{0}}^{t_{\mathrm{e}}} v^{T}(t) A_{1} v(t) \mathrm{d} t,
$$

for any $t_{\varrho}, t_{0} \in[0,+\infty)$ and $t_{0} \leq t_{\varrho}$, in which $S(t)=V_{1}(t) / 2$.
Theorem 3. Under the impulsive controller (9), the $M M D N N$ s (12) are output-strictly passive over $\mathbb{S}\left(v_{1}, v_{2}\right)$ if there exist matrices $0<F_{1} \in \mathbb{R}^{n N \times n N}, \quad 0<F_{2} \in \mathbb{R}^{n N \times n N}$, $A \in \mathbb{R}^{\epsilon N \times n N}$, and $0<A_{2} \in \mathbb{R}^{\epsilon N \times \epsilon N}$ and a scalar $\gamma \in(0,1]$ such that

$$
\begin{gathered}
\Gamma_{3 r h}=\left(\begin{array}{cc}
\Omega_{5 r h} & \Omega_{6 r} \\
\Omega_{6 r}^{T} & \Omega_{7}
\end{array}\right) \leq 0, \\
\left(\begin{array}{cc}
-\gamma F_{1} & \left(I_{n N}+B \otimes \Phi\right)^{T} F_{2} \\
F_{2}\left(I_{n N}+B \otimes \Phi\right) & -F_{2}
\end{array}\right) \leq 0,
\end{gathered}
$$

in which $r=1,2, h=1,2, \Omega_{5 r h}=1 / \nu_{h}\left(F_{1}-F_{2}\right)-F_{r}\left(I_{N} \otimes\right.$ $D)-\left(I_{N} \otimes D\right) F_{r}+\sum_{l=1}^{p}\left\{F_{r}\left[I_{N} \otimes\left(W_{l} W_{l}^{T}\right)\right] F_{r}+\left(I_{N} \otimes \Lambda_{l}\right)\right\}+$ $2\left(I_{N} \otimes C_{1}^{T}\right) A_{2}\left(I_{N} \otimes C_{1}\right), \quad \Omega_{6 r}=2\left(I_{N} \otimes C_{1}^{T}\right) \quad A_{2}\left(I_{N} \otimes C_{2}\right)+$ $F_{r}-\left(I_{N} \otimes C_{1}^{T}\right) A$, and $\Omega_{7}=-A^{T}\left(I_{N} \otimes C_{2}\right)-\left(I_{N} \otimes C_{2}^{T}\right) A+$ $2\left(I_{N} \otimes C_{2}^{T}\right) A_{2}\left(I_{N} \otimes C_{2}\right)$.

Proof. Let $F(t)=\sum_{r=1}^{2} \phi_{r}(t) F_{r}, \Omega_{5}(t)=-\left(I_{N} \otimes D\right) F(t)+\phi$ $(t)\left(F_{1}-F_{2}\right)+\sum_{l=1}^{p}\left\{F(t)\left[I_{N} \otimes\left(W_{l} W_{l}^{T}\right)\right] F(t)+I_{N} \otimes \Lambda_{l}\right\}+2$ $\left(I_{N} \otimes C_{1}^{T}\right) A_{2}\left(I_{N} \otimes C_{1}\right)-F(t)\left(I_{N} \otimes D\right), \Omega_{6}(t)=2\left(I_{N} \otimes C_{1}^{T}\right)$ $A_{2}\left(I_{N} \otimes C_{2}\right)+F(t)-\left(I_{N} \otimes C_{1}^{T}\right) A$.

Then, it is derived from (36) that

$$
\Gamma_{3}(t) \triangleq \sum_{r, h=1}^{2} \phi_{r}(t) \psi_{h}(t) \Gamma_{3 r h}=\left(\begin{array}{cc}
\Omega_{5}(t) & \Omega_{6}(t) \\
\Omega_{6}^{T}(t) & \Omega_{7}
\end{array}\right) \leq 0 .
$$

Selecting the same $V_{1}$ as (17) for the networks (12) and utilizing (38), we get

$$
\begin{aligned}
\dot{V}_{1}( & t)-2 y^{T}(t) A v(t)+2 y^{T}(t) A_{2} y(t) \\
\leq & \zeta^{T}(t)\left\{\phi(t)\left(F_{1}-F_{2}\right)-\left(I_{N} \otimes D\right) F(t)-F(t)\left(I_{N} \otimes D\right)+2\left(I_{N} \otimes C_{1}^{T}\right) A_{2}\left(I_{N} \otimes C_{1}\right)+\sum_{\imath=1}^{p}\left\{I_{N} \otimes \Lambda_{\iota}+F(t) \times\left[I_{N} \otimes\left(W_{\iota} W_{\iota}^{T}\right)\right] F(t)\right\}\right\} \zeta(t) \\
& +2 \zeta^{T}(t)\left[2\left(\left(I_{N} \otimes C_{1}^{T}\right)\right)\left(A_{2}\left(I_{N} \otimes C_{2}\right)+F(t)-\left(I_{N} \otimes C_{1}^{T}\right) A\right] v(t)\right] \\
& +v^{T}(t)\left[-A^{T}\left(I_{N} \otimes C_{2}\right)-\left(I_{N} \otimes C_{2}^{T}\right) A+2\left(I_{N} \otimes C_{2}^{T}\right) A_{2}\left(I_{N} \otimes C_{2}\right)\right] v(t) \\
= & \Phi_{1}^{T}(t)\left(\begin{array}{cc}
\Omega_{5}(t) & \Omega_{6}(t) \\
\Omega_{6}^{T}(t) & \Omega_{7}
\end{array}\right) \varpi_{1}(t) \\
\leq & 0, t \in\left(t_{k}, t_{k+1}\right), \quad \mathbb{N} \ni k
\end{aligned}
$$

in which $\Phi_{1}(t)=\left(\zeta^{T}(t), v^{T}(t)\right)^{T}$.

Integrating (39) with respect to $t$ from $t_{0}$ to $t_{\mathrm{Q}}\left(t_{\eta} \leq t_{\mathrm{Q}}<t_{\eta+1}, \eta=0,1, \ldots\right)$, one has

$$
\begin{gathered}
\int_{t_{0}}^{t_{\mathrm{e}}}\left[\dot{V}_{1}(t)-2 y^{T}(t) A v(t)+2 y^{T}(t) A_{2} y(t)\right] \mathrm{d} t \\
=\left[V_{1}\left(t_{\mathrm{e}}\right)-V_{1}\left(t_{0}\right)\right]+\sum_{\sigma=1}^{\eta}\left[V_{1}\left(t_{\sigma}^{-}\right)-V_{1}\left(t_{\sigma}\right)\right] \\
-2 \int_{t_{0}}^{t_{\mathrm{e}}}\left[y^{T}(t) A v(t)-y^{T}(t) A_{2} y(t)\right] \mathrm{d} t,
\end{gathered}
$$

in which $t_{\mathrm{\rho}}, t_{0} \in[0,+\infty)$ and $t_{0} \leq t_{\mathrm{e}}$.

At the impulse time $t_{k}, k \in \mathbb{N}$, according to the definition of $F(t)$, we derive

$$
\begin{aligned}
& F\left(t_{k}^{-}\right)=F_{1}, \\
& F\left(t_{k}\right)=F_{2} .
\end{aligned}
$$

It is obtained from (12), (17), and (37) that

$$
V_{1}\left(t_{k}\right) \leq V_{1}\left(t_{k}^{-}\right), \quad \mathbb{N} \ni k .
$$

Using (39)-(42), we can acquire 
$V_{1}\left(t_{\varrho}\right)-V_{1}\left(t_{0}\right) \leq 2 \int_{t_{0}}^{t_{\mathrm{e}}}\left[y^{T}(t) A v(t)-y^{T}(t) A_{2} y(t)\right] \mathrm{d} t$.

Therefore,

$S\left(t_{\varrho}\right)-S\left(t_{0}\right) \leq \int_{t_{0}}^{t_{\mathrm{e}}} y^{T}(t) A v(t) \mathrm{d} t-\int_{t_{0}}^{t_{\mathrm{e}}} y^{T}(t) A_{2} y(t) \mathrm{d} t$,

for any $t_{\mathrm{\rho}}, t_{0} \in[0,+\infty)$ and $t_{0} \leq t_{\mathrm{\rho}}$, in which $S(t)=V_{1}(t) / 2$.

Remark 1. In recent years, as an effective method to study synchronization, the passivity of NNs has been investigated by some researchers [12-17]. Impulsive control is a popular control method among control methods due to its reliability, low cost, and flexibility [22]. Using impulsive control strategies, some authors have focused on the passivity and synchronization of NNs. However, the passivity and synchronization of MMDNNs have not been considered under impulsive control.

\section{Synchronization of MMDNNs via Impulsive Control}

Setting $v_{i}(t)=0$ in the MMDNNs (6), we obtain

$$
\dot{z}_{i}(t)=-D z_{i}(t)+\sum_{\imath=1}^{p} W_{\iota} g^{l}\left(z_{i}\left(t-\tau_{\iota}\right)\right)+J, \quad i \in \mathcal{N} .
$$

The initial value of (45) is given by

$$
z_{i}(t)=\chi_{i}(t), t \in[-\tau, 0], \quad i \in \mathcal{N},
$$

where $\chi_{i}(t) \in C\left([-\tau, 0], \mathbb{R}^{n}\right)$ is the set of continuous functions from $[-\tau, 0]$ to $\mathbb{R}^{n}, \chi(t)=\left(\chi_{1}^{T}(t), \chi_{2}^{T}(t), \ldots, \chi_{N}^{T}(t)\right)^{T}$.

Suppose that $z^{*}=\left(z_{1}^{*}, z_{2}^{*}, \ldots, z_{n}^{*}\right)^{T} \in \mathbb{R}^{n}$ is an equilibrium solution of an isolated node of the MMDNNs (45). Then, one has

$$
-D z^{*}+J+\sum_{\imath=1}^{p} W_{\iota} g^{\iota}\left(z^{*}\right)=0 .
$$

For the MMDNNs (45), select the following impulsive controller:

$$
u_{i}\left(t_{k}\right)=\sum_{j=1}^{N} B_{i j} \Phi z_{j}\left(t_{k}^{-}\right), \quad k \in \mathbb{N}, i \in \mathcal{N},
$$

in which $B_{i j}$ and $\Phi$ have the same meanings as in the third section. Assume $z_{i}\left(t_{k}^{-}\right)=\lim _{t \longrightarrow t_{-}^{-}} z_{i}(t)$.

It is obtained from (45) and (48) that

$$
\left\{\begin{array}{l}
\dot{z}_{i}(t)=-D z_{i}(t)+\sum_{i=1}^{p} W_{\iota} g^{l}\left(z_{i}\left(t-\tau_{\iota}\right)\right)+J, \quad t \neq t_{k}, \\
I_{k}\left(z_{i}(t)\right)=\sum_{j=1}^{N} B_{i j} \Phi z_{j}\left(t_{k}^{-}\right), \quad k \in \mathbb{N}, i \in \mathcal{N},
\end{array}\right.
$$

where $I_{k}\left(z_{i}(t)\right)=z_{i}\left(t_{k}^{+}\right)-z_{i}\left(t_{k}^{-}\right), \quad z_{i}\left(t_{k}^{+}\right)=\lim _{t \rightarrow t_{k}^{+}} z_{i}(t)$. Suppose $z_{i}\left(t_{k}\right)=z_{i}\left(t_{k}^{+}\right)$.

Let $\zeta_{i}(t)=z_{i}(t)-z^{*}$. Then, it is found from (47) and (49) that

$$
\left\{\begin{array}{l}
\dot{\zeta}_{i}(t)=-D \zeta_{i}(t)+\sum_{i=1}^{p} W_{\iota}\left[g^{l}\left(z_{i}\left(t-\tau_{\iota}\right)\right)-g^{\imath}\left(z^{*}\right)\right], \quad t \neq t_{k}, \\
I_{k}\left(\zeta_{i}(t)\right)=\sum_{j=1}^{N} B_{i j} \Phi \zeta_{j}\left(t_{k}^{-}\right), \quad k \in \mathbb{N}, i \in \mathcal{N} .
\end{array}\right.
$$

Definition 3 (see [20]). The MMDNNs (45) achieve synchronization if

$$
\lim _{t \longrightarrow+\infty}\left\|z_{i}(t)-z^{*}\right\|=0, \quad i \in \mathcal{N} .
$$

Theorem 4. If there exist matrices $0<M_{1} \in \mathbb{R}^{n \times n}$ and $0<M_{2} \in \mathbb{R}^{n \times n}$ and scalars $\beta \in(0,1], \xi>0$ such that

$$
\begin{gathered}
\Gamma_{4 r h}=\left(\begin{array}{ccccc}
\Omega_{8 r h} & \Psi_{r 1} & \Psi_{r 2} & \cdots & \Psi_{r p} \\
\Psi_{r 1}^{T} & -I_{n} & 0 & \cdots & 0 \\
\Psi_{r 2}^{T} & 0 & -I_{n} & \cdots & 0 \\
\vdots & \vdots & \vdots & \ddots & \vdots \\
\Psi_{r p}^{T} & 0 & 0 & \cdots & -I_{n}
\end{array}\right) \leq 0, \\
\left(\begin{array}{ccc}
-\beta\left(I_{N} \otimes M_{1}\right) & & Y_{1} \\
Y_{1}^{T} & -\left(I_{N} \otimes M_{2}\right)
\end{array}\right) \leq 0,
\end{gathered}
$$

where $r=1,2, h=1,2, \Omega_{8 r h}=\left(\xi+\left(\ln \beta / v_{h}\right)\right) M_{r}-M_{r} \quad D-$ $D M_{r}+1 / \nu_{h}\left(M_{1}-M_{2}\right)+\sum_{l=1}^{p} e^{\xi_{\tau}} \Lambda_{l}, \quad \Psi_{r 1}=\beta^{-(1 / 2)} M_{r} W_{1}$, $\Psi_{r 2}=\beta^{-(1 / 2)} M_{r} W_{2}, \ldots, \Psi_{r p}=\beta^{-(1 / 2)} M_{r} W_{p}$, and $Y_{1}=$ $\left(I_{n N}+B \otimes \Phi\right)^{T}\left(I_{N} \otimes M_{2}\right), i \in \mathcal{N}$, then under the impulsive controller (48), the MMDNNs (45) achieve global exponential synchronization over $\mathbb{S}\left(\nu_{1}, v_{2}\right)$ with convergence rate $\xi / 2$.

Proof. Let $M(t)=\sum_{r=1}^{2} \phi_{r}(t) M_{r}, \Psi_{1}(t)=\beta^{-(1 / 2)} M(t) W_{1}$, $\Psi_{2}(t)=\beta^{-(1 / 2)} M(t) W_{2}, \ldots, \Psi_{p}(t)=\beta^{-(1 / 2)} M(t) W_{p}, \Omega_{8}(t)=$ $-M(t) D-D M(t)+[\xi+\phi(t) \ln \beta] M(t)+\sum_{t=1}^{p} e^{\xi \tau} \Lambda_{t}+\phi(t)$ $\left(M_{1}-M_{2}\right)$.

Then, by (52), we have

$$
\begin{aligned}
\Gamma_{4}(t) & \triangleq \sum_{r, h=1}^{2} \phi_{r}(t) \psi_{h}(t) \Gamma_{4 r h} \\
& =\left(\begin{array}{ccccc}
\Omega_{8}(t) & \Psi_{1}(t) & \Psi_{2}(t) & \cdots & \Psi_{p}(t) \\
\Psi_{1}^{T}(t) & -I_{n} & 0 & \cdots & 0 \\
\Psi_{2}^{T}(t) & 0 & -I_{n} & \cdots & 0 \\
\vdots & \vdots & \vdots & \ddots & \vdots \\
\Psi_{p}^{T}(t) & 0 & 0 & \cdots & -I_{n}
\end{array}\right) \leq 0 .
\end{aligned}
$$

The impulse-time-dependent Lyapunov functional for the MMDNNs (50) is considered as follows: 


$$
\begin{aligned}
V_{2}(t)= & \sum_{i=1}^{p} \sum_{i=1}^{N} \int_{t-\tau_{\iota}}^{t} e^{\xi\left(s+\tau-t_{0}\right)} \varphi(s) \zeta_{i}^{T}(s) \Lambda_{l} \zeta_{i}(s) \mathrm{d} s \\
& +\sum_{i=1}^{N} e^{\xi\left(t-t_{0}\right)} \varphi(t) \zeta_{i}^{T}(t) M(t) \zeta_{i}(t),
\end{aligned}
$$

in which $\varphi(t)=\beta^{\phi_{1}(t)}$.

For $\left(t_{k}, t_{k+1}\right) \ni t, \mathbb{N} \ni k$, differentiating $V_{2}(t)$ along the solution of the MMDNNs (50) and applying the fact that $\varphi(t-s) / \varphi(t) \geq \beta$, for all $0 \leq s$ and $t_{0}<t$, one gets

$$
\begin{aligned}
& \dot{V}_{2}(t)=\sum_{i=1}^{N} e^{\xi\left(t-t_{0}\right)} \varphi(t)\left\{\zeta_{i}^{T}(t)\left[(\xi+\phi(t) \ln \beta) M(t)+\phi(t)\left(M_{1}-M_{2}\right)-M(t) D-D M(t)\right] \zeta_{i}(t)\right. \\
& \left.+2 \sum_{\imath=1}^{p} M(t) W_{\iota}\left[g^{\imath}\left(z_{i}\left(t-\tau_{\imath}\right)\right)-g^{\imath}\left(\widehat{z}^{*}\right)\right]\right\} \\
& +\sum_{\imath=1}^{p} \sum_{i=1}^{N} e^{\xi\left(t+\tau-t_{0}\right)} \varphi(t) \zeta_{i}^{T}(t) \Lambda_{\iota} \zeta_{i}(t)-\sum_{\imath=1}^{p} \sum_{i=1}^{N} e^{\xi\left(t-\tau_{\iota}+\tau-t_{0}\right)} \varphi\left(t-\tau_{\iota}\right) \times \zeta_{i}^{T}\left(t-\tau_{\iota}\right) \Lambda_{\iota} \zeta_{i}\left(t-\tau_{\iota}\right) \\
& \leq \sum_{i=1}^{N} e^{\xi\left(t-t_{0}\right)} \varphi(t)\left\{\zeta_{i}^{T}(t)\left[(\xi+\phi(t) \ln \beta) M(t)+\phi(t)\left(M_{1}-M_{2}\right)+\sum_{l=1}^{p} e^{\xi \tau} \Lambda_{\iota}-M(t) D-D M(t)\right] \zeta_{i}(t)\right. \\
& \left.-\beta \sum_{\imath=1}^{p} \zeta_{i}^{T}\left(t-\tau_{\iota}\right) \Lambda_{\iota} \zeta_{i}\left(t-\tau_{\iota}\right)+2 \sum_{\imath=1}^{p} M(t) W_{\iota}\left[g^{\imath}\left(z_{i}\left(t-\tau_{\iota}\right)\right)-g^{\imath}\left(\widehat{z}^{*}\right)\right]\right\} .
\end{aligned}
$$

Moreover,

$$
\begin{aligned}
2 \zeta_{i}^{T} & (t) M(t) W_{\iota}\left[g^{\iota}\left(z_{i}\left(t-\tau_{\iota}\right)\right)-g^{\iota}\left(\widehat{z}^{*}\right)\right] \\
& \leq \beta^{-1} \zeta_{i}^{T}(t) M(t) W_{\iota} W_{\iota}^{T} M(t) \zeta_{i}(t) \\
& +\beta \zeta_{i}^{T}\left(t-\tau_{\iota}\right) \Lambda_{\iota} \zeta_{i}\left(t-\tau_{\iota}\right) .
\end{aligned}
$$

$$
\begin{aligned}
& \dot{V}_{2}(t) \leq \sum_{i=1}^{N} e^{\xi\left(t-t_{0}\right)} \varphi(t) \zeta_{i}^{T}(t)\left\{(\xi+\phi(t) \ln \beta) M(t)+\phi(t)\left(M_{1}-M_{2}\right)\right. \\
&\left.\quad-D M(t)-M(t) D+\sum_{\imath=1}^{p}\left[e^{\xi \tau} \Lambda_{\iota}+\beta^{-1} M(t) W_{\iota} W_{l}^{T} M(t)\right]\right\} \zeta_{i}(t) \\
& \leq 0, t \in\left(t_{k}, t_{k+1}\right), \quad \mathbb{N} \ni k .
\end{aligned}
$$

Therefore,

$$
V_{2}(t) \leq V_{2}\left(t_{k}\right), \forall t \in\left(t_{k}, t_{k+1}\right), \quad \mathbb{N} \ni k
$$

At the impulse time $t_{k}, k \in \mathbb{N}$, on the basis of the definitions of $M(t)$ and $\varphi(t)$, one has

$$
\begin{aligned}
\varphi\left(t_{k}^{-}\right) & =\beta, \\
\varphi\left(t_{k}\right) & =1, \\
M\left(t_{k}^{-}\right) & =M_{1}, \\
M\left(t_{k}\right) & =M_{2} .
\end{aligned}
$$

Combining (50), (53), and (55) together, we derive that 


$$
\begin{aligned}
& V_{2}\left(t_{k}\right)=\sum_{i=1}^{N} e^{\xi\left(t_{k}-t_{0}\right)} \varphi\left(t_{k}\right) \zeta_{i}^{T}\left(t_{k}\right) M\left(t_{k}\right) \zeta_{i}\left(t_{k}\right) \\
& +\sum_{\imath=1}^{p} \sum_{i=1}^{N} \int_{t_{k}-\tau_{\iota}}^{t_{k}} e^{\xi\left(s+\tau-t_{0}\right)} \varphi(s) \zeta_{i}^{T}(s) \Lambda_{\iota} \zeta_{i}(s) \mathrm{d} s \\
& =e^{\xi\left(t_{k}-t_{0}\right)} \varphi\left(t_{k}\right) \zeta^{T}\left(t_{k}\right)\left(I_{N} \otimes M\left(t_{k}\right)\right) \zeta\left(t_{k}\right) \\
& +\sum_{l=1}^{p} \sum_{i=1}^{N} \int_{t_{k}-\tau_{l}}^{t_{k}} e^{\xi\left(s+\tau-t_{0}\right)} \varphi(s) \zeta_{i}^{T}(s) \Lambda_{\iota} \zeta_{i}(s) \mathrm{d} s \\
& =e^{\xi\left(t_{k}-t_{0}\right)} \zeta^{T}\left(t_{k}^{-}\right)\left(I_{n N}+B \otimes \Phi\right)^{T}\left(I_{N} \otimes M_{2}\right) \\
& \left(I_{n N}+B \otimes \Phi\right) \zeta\left(t_{k}^{-}\right) \\
& +\sum_{i=1}^{p} \sum_{i=1}^{N} \int_{t_{k}-\tau_{l}}^{t_{k}} e^{\xi\left(s+\tau-t_{0}\right)} \varphi(s) \zeta_{i}^{T}(s) \Lambda_{\iota} \zeta_{i}(s) \mathrm{d} s \\
& \leq e^{\xi\left(t_{k}-t_{0}\right)} \beta \zeta^{T}\left(t_{k}^{-}\right)\left(I_{N} \otimes M_{1}\right) \zeta\left(t_{k}^{-}\right) \\
& +\sum_{i=1}^{p} \sum_{i=1}^{N} \int_{t_{k}-\tau_{l}}^{t_{k}} e^{\xi\left(s+\tau-t_{0}\right)} \varphi(s) \zeta_{i}^{T}(s) \Lambda_{\iota} \zeta_{i}(s) d s \\
& =\sum_{i=1}^{N} e^{\xi\left(t_{k}-t_{0}\right)} \varphi\left(t_{k}^{-}\right) \zeta_{i}^{T}\left(t_{k}^{-}\right) M\left(t_{k}^{-}\right) \zeta_{i}\left(t_{k}^{-}\right) \\
& +\sum_{i=1}^{p} \sum_{i=1}^{N} \int_{t_{k}-\tau_{\iota}}^{t_{k}} e^{\xi\left(s+\tau-t_{0}\right)} \varphi(s) \zeta_{i}^{T}(s) \Lambda_{\iota} \zeta_{i}(s) \mathrm{d} s \\
& =V_{2}\left(t_{k}^{-}\right), \quad \mathbb{N} \ni k \text {. }
\end{aligned}
$$

By (59) and (61), one obtains $V_{2}\left(t_{0}\right) \geq V_{2}(t), \forall t_{0} \leq t$. Hence, we acquire

$$
\begin{aligned}
\|\zeta(t)\| \leq & \sqrt{\frac{\bar{\lambda}_{M}+\sum_{l=1}^{p} \lambda_{\max }\left(\Lambda_{\iota}\right)\left(e^{\xi \tau}-1\right) / \xi}{\beta \lambda_{M}}} \\
& \cdot\left\|\chi\left(t_{0}\right)-\widehat{z}^{*}\right\|_{\tau} e^{-(\xi / 2)\left(t-t_{0}\right)}, \quad t_{0} \leq t,
\end{aligned}
$$

in $\quad$ which $\quad \bar{\lambda}_{M}=\max \left\{\lambda_{\max }\left(M_{1}\right), \lambda_{\max }\left(M_{2}\right)\right\}, \quad \underline{\lambda}_{M}=$ $\min \left\{\lambda_{\min }\left(M_{1}\right), \lambda_{\min }\left(M_{2}\right)\right\}$. Thus, the MMDNNs (45) reach global exponential synchronization over $\mathbb{S}\left(\nu_{1}, v_{2}\right)$ with convergence rate $\xi / 2$.

Remark 2. Compared with [37], the sufficient condition for the synchronization of MMDNNs depends on the length of impulsive intervals, and the time-varying Lyapunov function in Theorem 4 can capture the dynamical characteristics of MMDNNs.

\section{Numerical Examples}

Example 1. The MMDNNs are given by

$$
\dot{z}_{i}(t)=-D z_{i}(t)+\sum_{\imath=1}^{3} W_{\iota} g^{t}\left(z_{i}\left(t-\tau_{\iota}\right)\right)+J+v_{i}(t),
$$

in which $i=1,2, \ldots, 5, g_{\varsigma}^{1}(\theta)=(1 / 8)(|\theta+1|-|\theta-1|), g_{\varsigma}^{2}(\theta)=$ $(1 / 16)(|\theta+1|-|\theta-1|), g_{\varsigma}^{3}(\theta)=(1 / 32)(|\theta+1|-|\theta-1|), \quad \varsigma=$
$1,2,3, J=(0,0,0)^{T}, D=\operatorname{diag}(0.9,0.8,0.9), \tau_{1}=0.3, \tau_{2}=0.5$, $\tau=\tau_{3}=0.7$,

$$
\begin{aligned}
& W_{1}=\left(\begin{array}{lll}
0.1 & 0.2 & 0.1 \\
0.3 & 0.1 & 0.3 \\
0.4 & 0.2 & 0.4
\end{array}\right), \\
& W_{2}=\left(\begin{array}{lll}
0.2 & 0.3 & 0.4 \\
0.1 & 0.4 & 0.5 \\
0.3 & 0.5 & 0.1
\end{array}\right), \\
& W_{3}=\left(\begin{array}{lll}
0.2 & 0.5 & 0.5 \\
0.4 & 0.3 & 0.3 \\
0.1 & 0.6 & 0.3
\end{array}\right) .
\end{aligned}
$$

Apparently, $g_{\varsigma}^{1}(\cdot), g_{\varsigma}^{2}(\cdot)$, and $g_{\varsigma}^{3},(\cdot),(\varsigma=1,2,3)$, respectively, satisfy the Lipschitz condition with $\rho_{\varsigma}^{1}=0.25, \rho_{\varsigma}^{2}=0.125$, and $\rho_{\varsigma}^{3}=0.0625 . z^{*}=(0,0,0)^{T} \in \mathbb{R}^{3}$ is an equilibrium solution of the MMDNNs (63).

Take $t_{k}=k, \Phi=\operatorname{diag}(0.2,0.3,0.1), v_{1}=1.12, v_{2}=2.12$, $\gamma=0.6$,

$$
\begin{aligned}
C_{1} & =\left(\begin{array}{lll}
0.4 & 0.3 & 0.2 \\
0.3 & 0.5 & 0.3 \\
0.2 & 0.1 & 0.1 \\
0.6 & 0.3 & 0.1
\end{array}\right), \\
C_{2} & =\left(\begin{array}{lll}
0.2 & 0.5 & 0.3 \\
0.4 & 0.1 & 0.4 \\
0.1 & 0.5 & 0.4 \\
0.2 & 0.1 & 0.3
\end{array}\right), \\
B & =\left(\begin{array}{ccccc}
-0.7 & 0.2 & 0 & 0.2 & 0.3 \\
0.1 & -0.4 & 0.1 & 0 & 0.2 \\
0 & 0.1 & -0.7 & 0.1 & 0.5 \\
0.1 & 0 & 0.2 & -0.4 & 0.1 \\
0.1 & 0.2 & 0.1 & 0.2 & -0.6
\end{array}\right) .
\end{aligned}
$$

Case 1. Utilizing MATLAB YALMIP Toolbox, one obtains

$$
\begin{aligned}
& F_{1}=I_{5} \otimes\left(\begin{array}{ccc}
0.3631 & -0.4413 & 0.4421 \\
-0.4413 & 2.1340 & -1.3911 \\
0.4421 & -1.3911 & 1.4273
\end{array}\right)>0, \\
& F_{2}=I_{5} \otimes\left(\begin{array}{ccc}
0.2043 & -0.2327 & 0.2396 \\
-0.2327 & 1.1282 & -0.7170 \\
0.2396 & -0.7170 & 0.7490
\end{array}\right)>0, \\
& A=I_{5} \otimes\left(\begin{array}{ccc}
-17.7983 & 82.4589 & -36.1229 \\
5.1034 & -28.0123 & 7.4528 \\
16.4980 & -40.5024 & 56.8256 \\
4.0410 & -26.6311 & 1.9003
\end{array}\right),
\end{aligned}
$$


satisfying (14) and (15). From Theorem 1, the MMDNNs (12) under the impulsive controller (9) are passive.

Case 2. Utilizing MATLAB YALMIP Toolbox, one gets

$$
\begin{aligned}
& F_{1}=I_{5} \otimes\left(\begin{array}{ccc}
0.3501 & -0.4108 & 0.4248 \\
-0.4108 & 2.0450 & -1.3180 \\
0.4248 & -1.3180 & 1.3634
\end{array}\right)>0, \\
& F_{2}=I_{5} \otimes\left(\begin{array}{ccc}
0.1991 & -0.2203 & 0.2343 \\
-0.2203 & 1.0979 & -0.6921 \\
0.2343 & -0.6921 & 0.7281
\end{array}\right)>0, \\
& A=I_{5} \otimes\left(\begin{array}{ccc}
-37.2329 & 83.8487 & -129.0016 \\
10.8454 & -29.4624 & 34.1967 \\
33.2126 & -33.9720 & 141.5292 \\
8.5271 & -28.8543 & 22.1856
\end{array}\right), \\
& A_{1}=I_{5} \otimes\left(\begin{array}{ccc}
1.1069 & -0.3548 & 5.0976 \\
-0.3548 & 4.8449 & 1.3080 \\
5.0976 & 1.3080 & 25.3992
\end{array}\right),
\end{aligned}
$$

satisfying (27) and (28). From Theorem 2, the MMDNNs (12) under the impulsive controller (9) are input-strictly passive.

Case 3. Utilizing MATLAB YALMIP Toolbox, one derives

$$
\begin{aligned}
& F_{1}=I_{5} \otimes\left(\begin{array}{ccc}
0.3936 & -0.4735 & 0.4538 \\
-0.4735 & 2.2493 & -1.4582 \\
0.4538 & -1.4582 & 1.4735
\end{array}\right)>0 \text {, } \\
& F_{2}=I_{5} \otimes\left(\begin{array}{ccc}
0.2258 & -0.2531 & 0.2484 \\
-0.2531 & 1.2036 & -0.7613 \\
0.2484 & -0.7613 & 0.7839
\end{array}\right)>0 \text {, } \\
& A=I_{5} \otimes\left(\begin{array}{ccc}
17.2060 & 97.5401 & -23.8482 \\
4.7611 & -33.8467 & 2.1733 \\
17.1505 & -43.0380 & 58.5388 \\
3.6457 & -32.6881 & -3.9551
\end{array}\right) \text {, } \\
& A_{2}=I_{5} \otimes\left(\begin{array}{cccc}
20.6353 & -7.8392 & -4.8377 & -7.9339 \\
-7.8392 & 3.4784 & -1.4360 & 3.7580 \\
-4.8377 & -1.4360 & 25.2331 & -4.0463 \\
-7.9339 & 3.7580 & -4.0463 & 4.5656
\end{array}\right)>0 \text {, }
\end{aligned}
$$

satisfying (36) and (37). According to Theorem 3, the MMDNNs (12) under the impulsive controller (9) are outputstrictly passive. Figure 1 shows the results of simulation.

Example 2. The MMDNNs are given by

$$
\dot{z}_{i}(t)=-D z_{i}(t)+\sum_{i=1}^{3} W_{\iota} g^{t}\left(z_{i}\left(t-\tau_{\iota}\right)\right)+J
$$

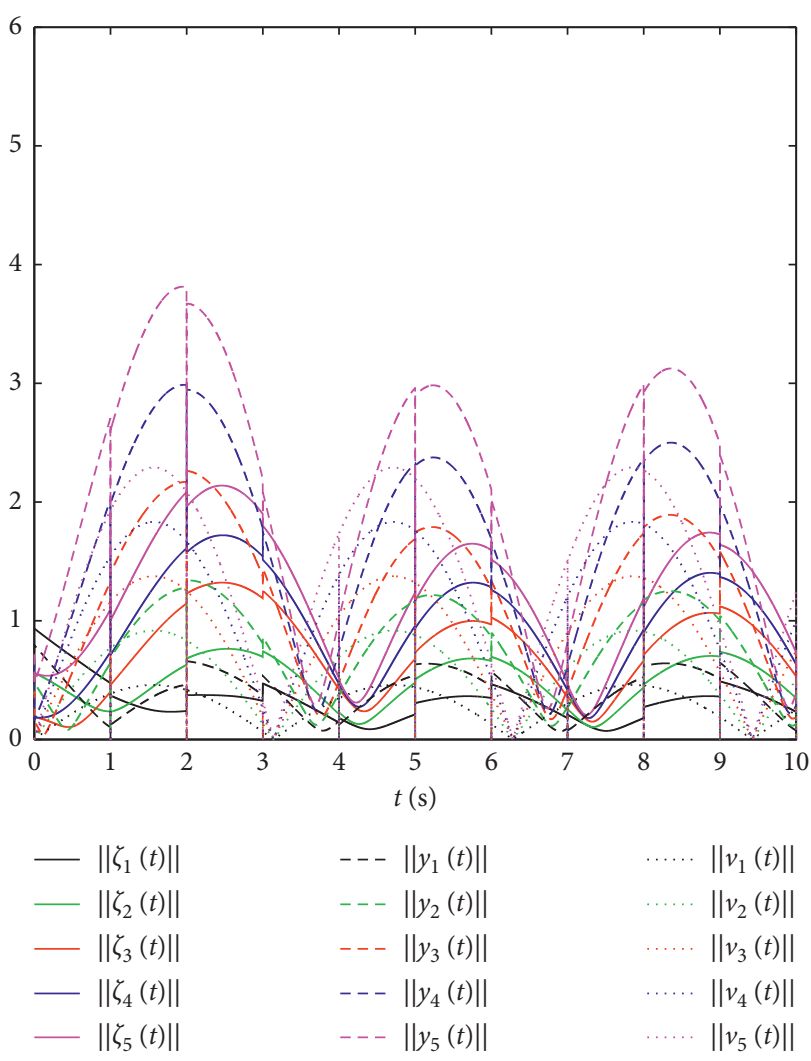

Figure 1: $\left\|\zeta_{i}(t)\right\|,\left\|y_{i}(t)\right\|,\left\|v_{i}(t)\right\|, i=1,2, \ldots, 5$.

in which $i=1,2, \ldots, 5, g_{\varsigma}^{1}(\theta)=(1 / 8)(|\theta+1|-|\theta-1|)$, $g_{\varsigma}^{2}(\theta)=(1 / 16)(|\theta+1|-|\theta-1|), g_{\varsigma}^{3}(\theta)=(1 / 32)(|\theta+1|-$ $|\theta-1|), \varsigma=1,2,3, D=\operatorname{diag}(0.7,0.9,0.8), J=(0,0,0)^{T}, \tau_{1}=$ $0.2, \tau_{2}=0.3, \tau=\tau_{3}=0.4$,

$$
\begin{aligned}
& W_{1}=\left(\begin{array}{lll}
0.3 & 0.4 & 0.5 \\
0.5 & 0.6 & 0.1 \\
0.2 & 0.3 & 0.2
\end{array}\right), \\
& W_{2}=\left(\begin{array}{lll}
0.5 & 0.6 & 0.2 \\
0.2 & 0.3 & 0.3 \\
0.1 & 0.2 & 0.5
\end{array}\right), \\
& W_{3}=\left(\begin{array}{lll}
0.2 & 0.1 & 0.3 \\
0.3 & 0.4 & 0.4 \\
0.4 & 0.5 & 0.6
\end{array}\right) .
\end{aligned}
$$

Apparently, $g_{\varsigma}^{1}(\cdot), g_{\varsigma}^{2}(\cdot)$, and $g_{\varsigma}^{3},(\cdot),(\varsigma=1,2,3)$, respectively, satisfy the Lipschitz condition with $\rho_{\varsigma}^{1}=0.25, \rho_{\varsigma}^{2}=0.125$, and $\rho_{\varsigma}^{3}=0.0625 . z^{*}=(0,0,0)^{T} \in \mathbb{R}^{3}$ is an equilibrium solution of the MMDNNs (69).

Let $\quad t_{k}=1.8 k, v_{1}=1.3, \quad \nu_{2}=3.1, \beta=0.8, \xi=0.2$, $\Phi=\operatorname{diag}(0.5,0.4,0.2)$, 


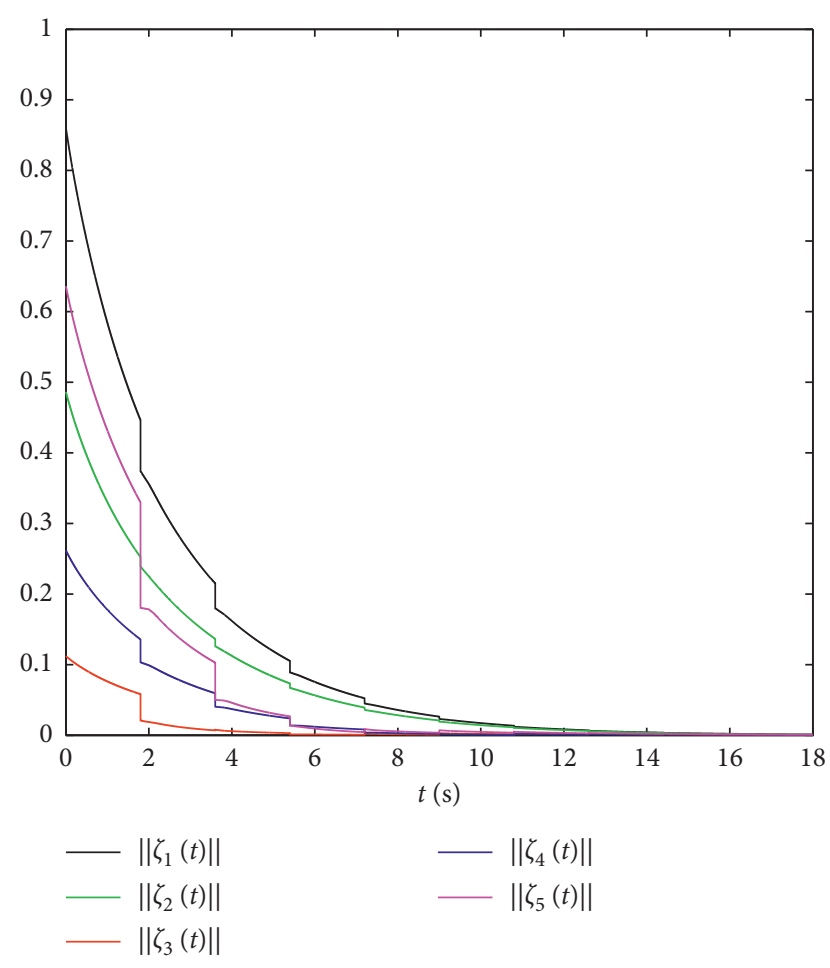

Figure $2:\left\|\zeta_{i}(t)\right\|, i=1,2, \ldots, 5$.

$$
B=\left(\begin{array}{ccccc}
-0.4 & 0.1 & 0 & 0.2 & 0.1 \\
0.3 & -0.6 & 0.2 & 0 & 0.1 \\
0 & 0.2 & -0.8 & 0.2 & 0.4 \\
0.2 & 0 & 0.1 & -0.5 & 0.2 \\
0.2 & 0.3 & 0.3 & 0.1 & -0.9
\end{array}\right) .
$$

By using MATLAB YALMIP Toolbox, we derive

$$
\begin{aligned}
& M_{1}=\left(\begin{array}{ccc}
1.1830 & -0.8947 & -0.2582 \\
-0.8947 & 2.9926 & -1.5950 \\
-0.2582 & -1.5950 & 2.0166
\end{array}\right)>0, \\
& M_{2}=\left(\begin{array}{ccc}
0.7928 & -0.5673 & -0.1779 \\
-0.5673 & 1.6871 & -0.7982 \\
-0.1779 & -0.7982 & 1.1134
\end{array}\right)>0,
\end{aligned}
$$

satisfying (52) and (53). According to Theorem 4, the MMDNNs (45) under the impulsive controller (48) are globally exponentially synchronized. Figure 2 shows the results of simulation.

\section{Conclusion}

In this paper, a class of multiple multi-delayed neural network models has been introduced, and the model can describe the dynamics of neurons more accurately. On the one hand, several passivity criteria for MMDNNs have been established by means of inequality techniques. On the other hand, the synchronization of the proposed model has also been researched based on stability theory. Finally, the correctness of the synchronization and passivity criteria has been verified by two numerical examples. In future work, we will design a suitable impulsive controller to further study the passivity and synchronization of multiple reaction-diffusion neural networks.

\section{Data Availability}

No data were used to support this study.

\section{Conflicts of Interest}

The authors declare that there are no conflicts of interest regarding the publication of this paper.

\section{Acknowledgments}

This study was supported in part by the Open Project Funded by the Chongqing Key Lab on IFBDA, School of Mathematical Sciences, Chongqing Normal University (CSSXKFKTQ201811), and in part by the National Natural Science Foundation of China under grant no. 11971081.

\section{References}

[1] J. Shin, K. Kwak, S. Kim, and H. J. Kim, “Adaptive range estimation in perspective vision system using neural networks," IEEE/ASME Transactions on Mechatronics, vol. 23, no. 2, pp. 972-977, 2018.

[2] J. Sun, G. Han, Z. Zeng, and Y. Wang, "Memristor-based neural network circuit of full-function pavlov associative memory with time delay and variable learning rate," IEEE Transactions on Cybernetics, vol. 50, no. 7, pp. 1-11, 2019.

[3] W.-R. Yang, C.-S. Wang, and C.-P. Chen, "Motion-pattern recognition system using a wavelet-neural network," IEEE Transactions on Consumer Electronics, vol. 65, no. 2, pp. 170-178, 2019.

[4] T. Dumas, A. Roumy, and C. Guillemot, "Context-adaptive neural network-based prediction for image compression," IEEE Transactions on Image Processing, vol. 29, pp. 679-693, 2020.

[5] X. M. Zhang and Q. L. Han, "Global asymptotic stability for a class of generalized neural networks with interval timevarying delays," IEEE Transactions on Neural Networks, vol. 22, no. 8, pp. 1180-1192, 2011.

[6] Z. Wang, H. Zhang, and B. Jiang, "LMI-based approach for global asymptotic stability analysis of recurrent neural networks with various delays and structures," IEEE Transactions on Neural Networks, vol. 22, no. 7, pp. 1032-1045, 2011.

[7] B. Zhang, J. Lam, and S. Xu, "Stability analysis of distributed delay neural networks based on relaxed Lyapunov-Krasovskii functionals," IEEE Transactions on Neural Networks and Learning Systems, vol. 26, no. 7, pp. 1480-1492, 2015.

[8] R. Samli, S. Senan, E. Yucel, and Z. Orman, "Some generalized global stability criteria for delayed Cohen-Grossberg neural networks of neutral-type," Neural Networks, vol. 116, pp. 198-207, 2019.

[9] Z. Li, H. Yan, H. Zhang, X. Zhan, and C. Huang, "Stability analysis for delayed neural networks via improved auxiliary polynomial-based functions," IEEE Transactions on Neural Networks and Learning Systems, vol. 30, no. 8, pp. 2562-2568, 2019. 
[10] R. Zhang, D. Zeng, J. H. Park, Y. Liu, and S. Zhong, "A new approach to stochastic stability of Markovian neural networks with generalized transition rates," IEEE Transactions on Neural Networks and Learning Systems, vol. 30, no. 2, pp. 499-510, 2019.

[11] C. $\mathrm{Hu}, \mathrm{H} . \mathrm{He}$, and H. Jiang, "Fixed/preassigned-time synchronization of complex networks via improving fixed-time stability," IEEE Transactions on Cybernetics, pp. 1-11, 2020.

[12] C. Li and X. Liao, "Passivity analysis of neural networks with time delay," IEEE Transactions on Circuits and Systems-II: Express Briefs, vol. 52, no. 8, pp. 471-475, 2005.

[13] S. Xu, W. Zheng, and Y. Zou, "Passivity analysis of neural networks with time-varying delays," IEEE Transactions on Circuits and Systems-II: Express Briefs, vol. 56, no. 4, pp. 325-329, 2009.

[14] J. Lian and J. Wang, "Passivity of switched recurrent neural networks with time-varying delays," IEEE Transactions on Neural Networks and Learning Systems, vol. 26, no. 2, pp. 357-366, 2015.

[15] Y. Chen, Z. Fu, Y. Liu, and F. E. Alsaadi, "Further results on passivity analysis of delayed neural networks with leakage delay," Neurocomputing, vol. 224, pp. 135-141, 2017.

[16] S. Saravanan, V. Umesha, M. Syed Ali, and S. Padmanabhan, "Exponential passivity for uncertain neural networks with time-varying delays based on weighted integral inequalities," Neurocomputing, vol. 314, pp. 429-436, 2018.

[17] Y. Cao, R. Samidurai, and R. Sriraman, "Robust passivity analysis for uncertain neural networks with leakage delay and additive time-varying delays by using general activation function," Mathematics and Computers in Simulation, vol. 155, pp. 57-77, 2019.

[18] X.-L. An, L. Zhang, Y.-Z. Li, and J.-G. Zhang, "Synchronization analysis of complex networks with multi-weights and its application in public traffic network," Physica A: Statistical Mechanics and its Applications, vol. 412, pp. 149-156, 2014.

[19] X.-L. An, L. Zhang, and J.-G. Zhang, "Research on urban public traffic network with multi-weights based on single bus transfer junction," Physica A: Statistical Mechanics and its Applications, vol. 436, pp. 748-755, 2015.

[20] J.-L. Wang, P.-C. Wei, H.-N. Wu, T. Huang, and M. Xu, "Pinning synchronization of complex dynamical networks with multiweights," IEEE Transactions on Systems, Man, and Cybernetics: Systems, vol. 49, no. 7, pp. 1357-1370, 2019.

[21] J.-L. Wang, M. Xu, H.-N. Wu, and T. Huang, "Passivity analysis and pinning control of multi-weighted complex dynamical networks," IEEE Transactions on Network Science and Engineering, vol. 6, no. 1, pp. 60-73, 2019.

[22] G. Wen, X. Zhai, Z. Peng, and A. Rahmani, "Fault-tolerant secure consensus tracking of delayed nonlinear multi-agent systems with deception attacks and uncertain parameters via impulsive control," Communications in Nonlinear Science and Numerical Simulation, vol. 82, Article ID 105043, 2020.

[23] C. Hu, H. Jiang, and Z. Teng, "Globally exponential stability for delayed neural networks under impulsive control," Neural Processing Letters, vol. 31, no. 2, pp. 105-127, 2010.

[24] X.-A. Li and J.-Z. Zou, "Globally exponential stability for stochastic delayed neural networks under impulsive control," Procedia Engineering, vol. 15, pp. 386-391, 2011.

[25] Q. Zhu and J. Cao, "Stability analysis of Markovian jump stochastic BAM neural networks with impulse control and mixed time delays," IEEE Transactions on Network Science and Engineering, vol. 23, no. 3, pp. 467-479, 2012.

[26] J. Qi, C. Li, and T. Huang, "Existence and exponential stability of periodic solution of delayed Cohen-Grossberg neural networks via impulsive control," Neural Computing and Applications, vol. 26, no. 6, pp. 1369-1377, 2015.

[27] H. Li, C. Li, and T. Huang, "Periodicity and stability for variable-time impulsive neural networks," Neural Networks, vol. 94, pp. 24-33, 2017.

[28] D. Li, P. Cheng, M. Hua, and F. Yao, "Robust exponential stability of uncertain impulsive stochastic neural networks with delayed impulses," Journal of the Franklin Institute, vol. 355, no. 17, pp. 8597-8618, 2018.

[29] L. Li and J. Jian, "Delay-dependent passivity analysis of impulsive neural networks with time-varying delays," Neurocomputing, vol. 168, pp. 276-282, 2015.

[30] R. Samidurai and R. Manivannan, "Robust passivity analysis for stochastic impulsive neural networks with leakage and additive time-varying delay components," Applied Mathematics and Computation, vol. 268, pp. 743-762, 2015.

[31] L. Zhou, "Delay-dependent and delay-independent passivity of a class of recurrent neural networks with impulse and multi-proportional delays," Neurocomputing, vol. 308, pp. 235-244, 2018.

[32] W. Jiang, G. Wen, Z. Peng, T. Huang, and A. Rahmani, "Fully distributed formation-containment control of heterogeneous linear multiagent systems," IEEE Transactions on Automatic Control, vol. 64, no. 9, pp. 3889-3896, 2019.

[33] J. Wang, L. Shen, J. Xia, Z. Wang, and X. Chen, "Asynchronous dissipative filtering for nonlinear jumping systems subject to fading channels," Journal of the Franklin Institute, vol. 357, no. 1, pp. 589-605, 2020.

[34] J. Wang, C. Yang, H. Shen, J. Cao, and L. Rutkowski, "Slidingmode control for slow-sampling singularly perturbed systems subject to Markov jump parameters," IEEE Transactions on Systems, Man, and Cybernetics: Systems, pp. 1-8, 2020.

[35] Y. Gong, G. Wen, Z. Peng, T. Huang, and Y. Chen, "Observerbased time-varying formation control of fractional-order multi-agent systems with general linear dynamics," IEEE Transactions on Circuits and Systems II: Express Briefs, vol. 67, no. 1, pp. 82-86, 2020.

[36] Y. Lv, C. Hu, J. Yu, H. Jiang, and T. Huang, "Edge-based fractional-order adaptive strategies for synchronization of fractional-order coupled networks with reaction-diffusion terms," IEEE Transactions on Cybernetics, vol. 50, no. 4, pp. 1582-1594, 2020.

[37] T. Wu, X. Huang, X. Chen, and J. Wang, "Sampled-data Ho exponential synchronization for delayed semi-Markov jump CDNs: a looped-functional approach," Applied Mathematics and Computation, vol. 377, Article ID 125156, 2020.

[38] C. Hu, H. He, and H. Jiang, "Synchronization of complexvalued dynamic networks with intermittently adaptive coupling: a direct error method," Automatica, vol. 112, Article ID 108675, 2020. 